\title{
Efficient Secret Sharing Without a Mutually Trusted Authority
}

\author{
Extended Abstract
}

\author{
Wen-Ai Jackson, Keith M. Martin* and Christine M. O'Keefe* \\ Department of Pure Mathematics, The University of Adelaide, Adelaide SA 5005, \\ Australia
}

\begin{abstract}
Traditional secret sharing schemes involve the use of a mutually trusted authority to assist in the generation and distribution of shares that will allow a secret to be protected among a set of participants. In contrast, this paper addresses the problem of establishing a secret sharing scheme for a given access structure without the use of a mutually trusted authority. A general protocol is discussed and several implementations of this protocol are presented. The efficiency of thcsc implementations is considered. The protocol is then refined and constructions are presented for mutually trusted authority free threshold schemes.
\end{abstract}

\section{Introduction}

A secret sharing scheme is a method by which a secret can be protected among a group of participants. Each participant holds a private share of the secret. Only certain sets of participants (authorised sets) are desired to be able to reconstruct the secret from their respective pooled shares. The collection of these subsets is the access structure of the secret sharing scheme. For the purposes of most of this paper, sets of participants that are not in the access structure (unauthorised sets) will not be able to determine any more information about the secret than is known publicly. Such schemes are often referred to as being perfect.

It is natural to make the assumption that if a set $A$ of participants contains a subset that belongs to an access structure then $A$ is itself in that access structure. We call access structures with this property monotone. A secret sharing scheme on $n$ participants in which precisely all subsets of size at least $k(1 \leq k \leq n)$ are in the access structure is known as a $(k, n)$-threshold scheme (we say that the access structure is $(k, n)$-lhreshold). Threshold schemes were the first types of secret sharing scheme proposed $([2,10])$.

We make a subtle distinction between two types of secret that can be protected by a secret sharing scheme. A secret is said to be explicit if it takes a fixed value that is predetermined by factors outside the secret sharing scheme design. In other words, the scheme is designed to protect a particular predetermined

\footnotetext{
* This work was supported by the Australian Research Council
} 
number within a given domain. This might be, say, a bank account number, the number of a security box or an enabling code. A secret is said to be implicit if it does not take a predetermined value. In this case the secret sharing scheme must protect a secret, but the value of the secret can be any number within a specified domain. A secret sharing scheme is more likely to have an implicit secret either in a situation where there is no obvious number associated with the secret, such as when the scheme is to be used to demonstrate concurrence in an access control protocol, or in a situation where the implicit secret value is $s u b$ sequently adopted as, say, a cryptographic key or the number of a secure vault. In these situations the implicit secret, and/or the shares that generate it, must be part of the initialisation of the device that verifies the secret. For instance, an application was described in [6] where the shares of an implicit secret were manually incorporated into the initialisation process of the locking mechanism of a vault door.

Traditional models for secret sharing schemes rely on the existence of a $M u$ tually Trusted Authority (MTA) to set up the scheme in the first place. This authority must be trusted by all the participants and can either be human (perhaps an organisation) or be a device. If the secret is explicit then the MTA is trusted with the knowledge of the explicit secret and with the generation and distribution of suitable shares that relate to the secret in question. In the case of an implicit secret, the MTA is further responsible for the generation of the implicit secret that is to be shared among the participants of the scheme.

We study here secret sharing schemes that do not require the existence of an MTA during their set-up protocols. We will thus refer to such schemes as being MTA-free. In an MTA-free scheme the participants generate their own shares. The MTA-free schemes that we consider all have implicit secrets. Unless there is a singleton participant set in the access structure of a secret sharing scheme, it does not seem very likely that a protocol can be devised which allows a group of participants to generate shares to protect an explicit secret. If there is a singleton participant set in the access structure then, since that participant effectively knows the secret directly from their share, that participant could (in theory) play the role of an MTA and generate shares of the (explicit) secret for the other participants. Indeed, a traditional secret sharing scheme can be thought of as a secret sharing scheme of this type where the MTA is an extra participant, in the access structure as a singleton set.

We note first that there does exist one family of monotone access structures which can be easily realised by MTA-free secret sharing schemes. A (unanimous) $(n, n)$-threshold scheme can be constructed without an MTA, as follows. Let $w$ be a fixed positive integer.

- Each participant chooses a (random) share from $\mathbf{Z}_{w}$;

- The (implicit) secret is the sum of the participants' shares modulo $w$.

The first paper to consider constructions of more general MTA-free schemes was by Meadows [9]. In this novel paper a $(k, n)$-threshold scheme was proposed which allows the first $k$ participants to generate their own (random) shares. Unfortunately a 'black box' is then required to generate the shares of the remaining 
$n-k$ participants. This black box must be trusted with the knowledge of all the shares and with the value of the (implicit) secret. Thus by our definition the black box is playing the role of an M'TA. The only possible advantage of this protocol is that the value of the implicit secret is directly determined from the shares chosen by the first $k$ participants. However this does not appear to be much different from a scheme set up by a (device-based) MTA that selects the implicit secret using a random number generator.

In 1991 Ingemarsson and Simmons [6] reconsidered the design of MTA-free schemes for general monotone access structures and suggested an elegant protocol. The basic idea of [6] is that the $n$ participants first generate shares of an (MTA-free) unanimous $(n, n)$-threshold scheme. The implicit secret of this unanimous scheme becomes the secret of the final scheme. Each participant then acts as their own M'TA and sets up a private secret sharing scheme to protect their share of the unanimous scheme among a number of the other participants. Thus a participant's share in the unanimous scheme becomes the explicit secret of their private sccret sharing scheme. It is quite possible that after carrying out this protocol, no participant will actually know the access structure of the induced secret sharing scheme. In [6] it is suggested that this procedure has the potential to realise an MTA-free scheme for any monotone access struclure. We will later prove this suggestion to be correct.

The main aim of this paper is to start with a monotone access structure $\Gamma$, and determine which initial MTA-free scheme and which private secret sharing schemes should be used in order to realise an MTA-free scheme for $\Gamma$. There is not necessarily a unique way of doing this and so we are particularly interested in trying to find efficient and economical methods. These are based on trying to minimise the number of shares that have to be generated, mutually communicated and stored by the participants in the scheme. In doing so we show that not all of the participants need to generate shares in the first instance. It is often the case that in an efficient realisation of an MTA-free scheme for $\Gamma$, some participants need only store shares that have been generated by other participants.

In Section 2 we discuss the concept of access structure domination, which is fundamental to the rest of the paper. Section 3 is about MTA-free schemes in general and includes a construction protocol that will work for any monotone access structure. In Section 4 we concentrate on MTA-free threshold schemes and show that some variations of the standard protocol can be used to improve scheme efficiency. We have been forced to omit most proofs in this extended abstract, but it is hoped that by way of examples we can illustrate the main ideas behind the constructions presented. Complete proofs will be provided in the full paper.

\section{Access Structure Domination}

Let $\Gamma$ be a monotone access structure defined on a participant set $\mathcal{P}$. The monotonicity of $\Gamma$ ensures that we can find a collection $\Gamma^{-}$of minimal authorised 
sets in $\Gamma$ and a set $\Gamma^{+}$of maximal unauthorised sets. Note that a participant need not belong to any minimal set in $\Gamma$. If every participant does belong to a minimal set then we say that $\Gamma$ is connected. We recall from [1] that $\Gamma$ can be considered as a logical expression with the participants being boolean variables. Let $I^{r-}=\left\{C_{1}, \ldots, C_{r}\right\}$, let + denote logical $O R$ and let juxtaposition denote logical AND. Then the disjunctive normal form of the logical equivalent of $T$ is $\Gamma=C_{1}+\cdots+C_{r}$. It follows that a subset $A$ of participants is in the access structure $\Gamma$ if and only if the logical equivalent of $\Gamma$ is true when the variables in $A$ are all true. For example, let $\mathcal{P}=\{a, b, c, d\}$ and $\Gamma^{-}=\{\{a, b, c\},\{c, d\}\}$. Then we write $\Gamma=a b c+c d$, or equivalently $\Gamma=(a b+d) c$.

We now recall from [8] a useful family of monotone access structures that can be derived from $\Gamma$. Let $A \subseteq \mathcal{P}$. We define the contraction $\Gamma \cdot A$ of $\Gamma$ at $A$ to be the monotone access structure on $\mathcal{P}$ given by

$$
B \in \Gamma \cdot A \Longleftrightarrow B \cup A \in \Gamma \text {. }
$$

Conceptually, $\Gamma \cdot A$ is the access structure that results if the shares belonging to the participants in $A$ are publicly revealed. For example, if $I=a b c+c d$ then $\Gamma \cdot c=a b+d$ and $\Gamma \cdot d=c$.

Now let $\Gamma_{0}$ be a monotone access structure defined on $\mathcal{P}=\left\{p_{1}, \ldots, p_{n}\right\}$. Associate with each $p_{i} \in \mathcal{P}$ a monotone access structure $\Gamma_{i}$ defined on $\mathcal{P}$. Let $\Gamma=\left(\Gamma_{0} ; \Gamma_{1}, \ldots, \Gamma_{n}\right)$ be the monotone access structure defined on $\mathcal{P}$ that is formed by replacing $p_{i}$ by $\Gamma_{i}$ in the logical equivalent of $\Gamma_{0}$.

Example 1. Let $\mathcal{P}=\{a, b, c, d\}$. Let $\Gamma_{0}=a b c d, \Gamma_{a}=c, \Gamma_{b}=c+d, \Gamma_{c}=d$ and $\Gamma_{d}=d$. Then $\Gamma=\left(\Gamma_{0} ; \Gamma_{a}, \Gamma_{b}, \Gamma_{c}, \Gamma_{d}\right)=c(c+d) d d=c d$. Similarly, if $\Gamma_{0}=a b c d$, $\Gamma_{a}=a+c, \Gamma_{b}=b+d, \Gamma_{c}=$ 'true' (in other words, $\left(\Gamma_{c}\right)^{-}=\{\emptyset\}$ ) and $\Gamma_{d}=$ 'true' then $\Gamma=\left(\Gamma_{0} ; \Gamma_{a}, \Gamma_{b}, \Gamma_{c}, \Gamma_{d}\right)=(a+c)(b+d)=a b+a d+b c+c d$.

For $A \subseteq \mathcal{P}$, let $X(A)=\left\{p_{i} \mid A \in \Gamma_{i}\right\}$. We can also describe $\Gamma=\left(\Gamma_{0} ; \Gamma_{1}, \ldots, \Gamma_{n}\right)$ in the following way.

Lemma 1. Let $\Gamma, \Gamma_{0}, \Gamma_{1}, \ldots, \Gamma_{n}$ be monolone access structures defined on $\mathcal{P}=$ $\left\{p_{1}, \ldots, p_{n}\right\}$. Then the following two statements are equivalent:

1. $\Gamma=\left(\Gamma_{0} ; \Gamma_{1}, \ldots, \Gamma_{n}\right)$;

2. For every $A \subseteq \mathcal{P}$ we have $A \in \Gamma$ if and only if $X(A) \in \Gamma_{0}$.

Now let $\Gamma_{0}$ and $\Gamma$ be distinct monotone access structures defined on $\mathcal{P}=$ $\left\{p_{1}, \ldots, p_{n}\right\}$. Using terminology suggested in [11], we say that $\Gamma_{0}$ dominates $\Gamma$ if there exist monotone access structures $\Gamma_{1}, \ldots, \Gamma_{n}$ such that

1. $\left\{p_{i}\right\} \in \Gamma_{i}$ (for each $\left.i, 1 \leq i \leq n\right)$;

2. $\Gamma=\left(\Gamma_{0} ; \Gamma_{1}, \ldots, \Gamma_{n}\right)$.

Thus, from Example 1, we see that $\Gamma_{0}=a b c d$ dominates $\Gamma=a b+a d+b c+$ $c d$. We say that $\Gamma_{0}$ directly dominates $\Gamma$ if there does not exist a monotone access structure $\Gamma^{\prime}$ (distinct from $\Gamma_{0}$ and $\Gamma$ ) such that $\Gamma_{0}$ dominates $\Gamma^{\prime}$ and $\Gamma^{\prime}$ dominates $\Gamma$. We now classify all the monotone access structures that are (directly) dominated by a given monotone access structure. 
Theorem 2. Let $\Gamma_{0}$ and $\Gamma$ be monotone access structures defined on $\mathcal{P}$. Then $\Gamma_{0}$ dominates $\Gamma$ if and only if $\Gamma_{0} \subseteq \Gamma$.

The next result is an interpretation of the main theorem in [11].

Result 3. Let $\Gamma_{0}$ and $\Gamma$ be monotone access structures defined on $\mathcal{P}$. Then $\Gamma_{0}$ directly dominates $\Gamma$ if and only if there exists a (unique) maximal unauthorised subset $B$ of $\Gamma_{0}$ such that $\Gamma=\Gamma_{0} \cup\{B\}$.

\section{Mutually Trusted Authority free Schemes}

We first give a basic model for secret sharing (see, for example, [13]). We will use the entropy function in our definition (see, for example, [5] for an introduction to entropy and its properties). Let $\mathcal{P}=\left\{p_{1}, \ldots, p_{n}\right\}$ be a participant set and let $s$ be a secret. Let participant $p_{i}$ receive a share from a set $\left[p_{i}\right]$ and let the secret come from a set [s]. A secret sharing scheme for $\Gamma$ is a probability distribution $\rho$ defined on a set of distribution rules $\Omega \subseteq\left[p_{1}\right] \times \cdots \times\left[p_{n}\right] \times[s]$ such that for $A \subseteq \mathcal{P}$,

1. if $A \in \Gamma$ then $H(s \mid A)=0$;

2. if $A \notin \Gamma$ then $H(s \mid A)>0$.

If it is the case that for each $A \notin \Gamma$ we have $H(s \mid A)=H(s)$ then the secret sharing scheme is said to be perfect. We call $H\left(p_{i}\right)$ the size of the share associated with $p_{i}$, and $H(s)$ the size of the secret. It can be seen (for example [13]) that in any perfect secret sharing scheme, if $p_{i} \in A$ for some minimal authorised set $A$ then $H\left(p_{i}\right) \geq H(s)$. If $H\left(p_{i}\right)=H(s)$ for all such $p_{i}$ then we say that the perfect secret sharing scheme and its access structure are ideal. We note $([2,10])$ that ideal $(k, n)$-threshold schemes can be found for all $1 \leq k \leq n$.

In a traditional secret sharing scheme, an MTA selects a distribution rule $\pi$ from $\Omega$ with probability $\rho(\pi)$ and then distributes the appropriate shares to the participants of the scheme. In an MTA-free scheme the participants indirectly select a (random) distribution rule through the generation of their own (random) shares.

Consider the following extension of the protocol in [6] for setting up an MTAfree scheme. Firstly, let a subset $\mathcal{P}_{0}$ of the participants in $\mathcal{P}$ generate shares of a perfect scheme $M_{0}$ for some access structure $\Gamma_{0}$. Let $x_{i}$ denote the share generated by $p_{i}\left(p_{i} \in \mathcal{P}_{0}\right)$. Then let each $p_{i} \in \mathcal{P}_{0}$ construct a private perfect secret sharing scheme $M_{i}$ for $\Gamma_{i}$ on $\mathcal{P}$ to protect the explicit secret $x_{i}$. Thus $x_{i}$ can be obtained by $p_{i}$ or by any authorised set in $\Gamma_{i}$. In the degenerate case where $\Gamma_{i}^{-}=$ $\{\emptyset\}, p_{i}$ publicly reveals (broadcasts) their share. Otherwise $p_{i}$ communicates the shares of $M_{i}$ to the participants included in $M_{i}$. This process creates a new perfect secret sharing schemc $M$ for access structure $\Gamma=\left(\Gamma_{0} ; \Gamma_{1}, \ldots, \Gamma_{n}\right)$, where for each $p_{i} \notin \mathcal{P}_{0}$ we take $\Gamma_{i}$ to be such that $I_{i}^{-}=\{\emptyset\}$ and thus for each $i$ $(1 \leq i \leq n),\left\{p_{i}\right\} \in \Gamma_{i}$. For the structure of the distribution rules of $M$ in terms of those of $M_{0}$ and $M_{i}$, we refer to $[8,14]$, where constructions of this type were fully described. 
Simmons [11] asked which access structures $\Gamma$ could be realised from $\Gamma_{0}$ in this manner. In other words, which access structures $\Gamma$ are dominated by $\Gamma_{0}$ ? Theorem 2 concisely answers this question by showing that these are precisely the access structures $\Gamma$ such that $\Gamma \supseteq \Gamma_{0}$. We approach the problem from another direction in this paper. Namely, given an access structure $\Gamma$, exactly which access structures $\Gamma_{0}, \Gamma_{1}, \ldots, \Gamma_{n}$ such that $\Gamma=\left(\Gamma_{0} ; \Gamma_{1}, \ldots, \Gamma_{n}\right)$ should be chosen in order to (efficiently) generate an MTA-free scheme for $\Gamma$ ?

\subsection{The Base Access Structure}

The first issue to be considered in the design of an MTA-free scheme for $\Gamma$ is which initial access structure $\Gamma_{0}$ should be chosen. We refer to $\Gamma_{0}$ as the base access structure. We assume that each $p \in \mathcal{P}_{0}$ independently generates a random share of $M_{0}$ from set $[p]$.

Theorem 4. Let $\Gamma_{0}$ be the access structure of a secret sharing scheme $M_{0}$ defined on $\mathcal{P}$ such that for each $p \in \mathcal{P}$ the share held by $p$ in $M_{0}$ is independently and randomly chosen from the set $[p]$. Then $\Gamma_{0}$ has a unique minimal authorised set $\mathcal{P}_{0}$.

If $M_{0}$ is a perfect secret sharing scheme then it follows from Theorem 4 that $M_{0}$ is a unanimous threshold scheme defined on $\mathcal{P}_{0}$, and thus that the participants in $\mathcal{P} \backslash \mathcal{P}_{0}$ need not generate shares in the initial stage of the protocol. Thus from Theorems 2 and 4 we have the following criteria for selection of the base access structure $\Gamma_{0}$.

$-\Gamma_{0} \subseteq \Gamma$

- $\Gamma_{0}$ is unanimous threshold on $\mathcal{P}_{0}\left(P_{0} \subseteq \mathcal{P}\right)$.

\subsection{Measures of Efficiency}

There are three parameters that we might want to minimise for reasons of economy and efficiency in an MTA-free scheme. These are the total size $g(\mathcal{P})$ of shares generated by the participants, the total size $c(\mathcal{P})$ of shares communicated by participants, and the total size $s(\mathcal{P})$ of shares stored by participants in the scheme. For a participant $p \in \mathcal{P}$ let $g(p)$ be the sum of the sizes of shares generated by $p, c(p)$ be the sum of the sizes of shares communicated by $p$ and $s(p)$ be the sum of the sizes of shares stored by $p$.

In the light of Section 3.1, we assume that $M_{0}$ is chosen to be ideal and hence the shares of $M_{0}$ (and thus the secrets of $M_{i}$, for $p_{i} \in \mathcal{P}_{0}$ ) all have the same size $h$. For the purposes of the discussion immediately following we will take $h$ as the size of a 'unit' share.

Let $p \in \mathcal{P}$. If $p \notin \mathcal{P}_{0}$ then $g(p)=c(p)=0$. Otherwise, if $p \in \mathcal{P}_{0}$ then $p$ generates one sharc $x_{p}$ of the initial scheme and then a number (possibly zero) of shares of a private scheme to protect $x_{p}$. These extra shares are communicated 
to some of the other participants. Thus if $p \in \mathcal{P}_{0}$ then $g(p)=1+c(p)$. Hence in total,

$$
g(\mathcal{P})=c(\mathcal{P})+\left|\mathcal{P}_{0}\right|
$$

A participant $p \in \mathcal{P}_{0}$ either keeps the share $x_{p}$ secure or broadcasts it and hence does not need to store it. Let $\mathcal{P}_{0}^{\prime}$ be the subset of $\mathcal{P}_{0}$ who store their shares. All shares of private schemes that are communicated to $p \in \mathcal{P}$ are stored securely. Hence we have in total,

$$
s(\mathcal{P})=c(\mathcal{P})+\left|\mathcal{P}_{0}^{\prime}\right|
$$

Then from (1) and (2) we have,

$$
g(\mathcal{P})=s(\mathcal{P})+\left|\mathcal{P}_{0} \backslash \mathcal{P}_{0}^{\prime}\right| ; \quad c(\mathcal{P})=s(\mathcal{P})-\left|\mathcal{P}_{0}^{\prime}\right|
$$

Thus from (3) we see that $s(\mathcal{P})$ alone is an effective measure of efficiency since $g(\mathcal{P})$ and $c(\mathcal{P})$ are directly proportional to $s(\mathcal{P})$. In the event that two different schemes have the same total storage $s(\mathcal{P})$ then $(3)$ suggests that a scheme which has a large value of $\left|\mathcal{P}_{0}^{\prime}\right|$ relative to $\left|\mathcal{P}_{0}\right|$ is preferable.

Minimising storage has been the most studied measure of efficiency for traditional secret sharing schemes (for example [3, 4, 8, 14]). Efficiency rates can be calculated from the contribution vector (or convec) of the scheme. This is the vector $\left(c_{1}, \ldots, c_{n}\right)=(1 / H(s))\left(H\left(p_{1}\right), \ldots, H\left(p_{n}\right)\right)$. For perfect secret sharing schemes the most common efficiency measures are the information rate, which is the minimum $1 / c_{i}(1 \leq i \leq n)$, and the average information rate, which is $n /\left(c_{1}+\cdots+c_{n}\right)$. For MTA-free schemes we see that the total storage $s(\mathcal{P})$ is $c_{1}+\cdots+c_{n}$. For simplicity, in this paper we only consider minimising the total storage, which is equivalent to maximising the average information rate.

\subsection{The Private Access Structures}

The problem of constructing an efficient MTA-free scheme for $\Gamma$ is thus the problem of selecting a base access structure $\Gamma_{0}$ (subject to the constraints of Section 3.1) and a collection of private access structures $\Gamma_{p}\left(p \in \mathcal{P}_{0}\right)$ in such a way that $s(\mathcal{P})$ is minimised. As a standard for comparison we use what we call the Basic construction. This is in effect the most 'obvious' way of constructing an MTA-free scheme for $\Gamma$. The Basic construction is used in the proof of Theorem 2.

\section{The Basic Construction}

$\Gamma_{0} \quad(n, n)$-threshold on $\mathcal{P}$

$\Gamma_{p} \quad p+I$ (for each $\left.p \in \mathcal{P}\right)$

$M_{0} \quad$ ideal unanimous threshold scheme on $\mathcal{P}$

$M_{p} \quad$ perfect scheme for $\Gamma_{p}$ (for each $p \in \mathcal{P}$ ) 
Example 2. Let $\mathcal{P}=\mathcal{P}_{0}=\{a, b, c, d\}$ and $\Gamma=a b+a c+b c d$. Applying the Basic construction gives $\Gamma_{0}=a b c d, \Gamma_{a}=a+b c d, \Gamma_{b}=b+a c, \Gamma_{\mathfrak{c}}=c+a b$ and $\Gamma_{d}=d+a b+a c$. Since $\Gamma_{a}, \Gamma_{b}, \Gamma_{c}, \Gamma_{d}$ are all ideal (see [12]) we can find ideal $M_{a}, M_{b}, M_{c}, M_{d}$ and thus a scheme $M$ for $\Gamma$ with convec $\left(c_{a}, c_{b}, c_{c}, c_{d}\right)=$ $(4,4,4,2)$ (for example, participant a generates one unit share, and receives one unit share from each of $b, c, d)$.

We show that, by applying contractions, the Basic construction can be quite considerably improved upon. We call this modified construction method the Contraction construction.

\begin{tabular}{cl}
\hline & \multicolumn{1}{c}{ The Contraction Construction } \\
$\Gamma_{0}$ & $(a, a)$-threshold on $\mathcal{P}_{0}$, for some $\mathcal{P}_{0}=\left\{p_{1}, \ldots, p_{a}\right\} \in \Gamma$ \\
$\Gamma_{1}$ & $p_{1}+\Gamma$ \\
$I_{2}$ & $p_{2}+\Gamma \cdot p_{1}$ \\
$\vdots$ & $\vdots$ \\
$\Gamma_{a}$ & $p_{a}+\Gamma \cdot p_{1} p_{2} \ldots p_{a-1}$ \\
$M_{0}$ & ideal unanimous threshold scheme on $A$ \\
$M_{i}$ & perfect scheme for $\Gamma_{i}$ (for each $\left.i, 1 \leq i \leq a\right)$
\end{tabular}

Example 3. Let $\mathcal{P}$ and $\Gamma$ be as in Example 2. Applying the Contraction construction with $\mathcal{P}_{0}=\{a, b\}$ and $\Gamma_{0}=a b$ gives $\Gamma_{a}=a+b c d, \Gamma_{b}=b+c$. Since $\Gamma_{a}, \Gamma_{b}$ are ideal (see [12]) we can find ideal $M_{a}, M_{b}$ and thus a scheme $M_{1}$ for $\Gamma$ with convec $(1,2,2,1)$. Alternatively, applying the Contraction construction with $\mathcal{P}_{0}=\{b, c, d\}$ and $\Gamma_{0}=b c d$ gives $\Gamma_{b}=b+a c, \Gamma_{c}=c+a, \Gamma_{d}=d+a$. Since $\Gamma_{b}, \Gamma_{c}, \Gamma_{d}$ are ideal (see [12]) we can find ideal $M_{b}, M_{c}, M_{d}$ and thus a scheme $M_{2}$ for $\Gamma$ with convec $(3,1,2,1)$. Both $M_{1}$ and $M_{2}$ are considerably more efficient in terms of total storage (and information rate) than the scheme $M$ constructed in Example 2. Scheme $M_{1}$ is slightly more efficient than $M_{2}$.

\section{Mutually Trusted Authority free Threshold Schemes}

We now consider the special case of realising an MTA-free $(k, n)$-threshold scheme. We assume that $k<n$ since the case $k=n$ was covered in Section 1. Let $\mathcal{P}=\left\{p_{1}, \ldots, p_{n}\right\}$, let $1 \leq k<n$ and let $\Gamma$ be $(k, n)$-threshold on $\mathcal{P}$. The most efficient MTA-free scheme for $\Gamma$ we have seen thus far is by the Contraction construction applied to a minimal authorised set of $\Gamma$. In this case,

- $\Gamma_{0}=(k, k)$-threshold on $\mathcal{P}_{0}=\left\{p_{1}, \ldots, p_{k}\right\}$

- $\Gamma_{i}=p_{i}+\left((k-i+1, n-i)\right.$-threshold on $\left.\left\{p_{i+1}, \ldots, p_{n}\right\}\right)$ (for each $\left.i, 1 \leq i \leq k\right)$.

Using ideal threshold schemes $M_{0}, M_{1}, \ldots, M_{a}$ we can calculate the convec for the resulting scheme $M$. Each $p_{i}(1 \leq i \leq k)$ stores their share of $M_{0}$ and receives 
one share from each of $p_{1}, \ldots, p_{i-1}$. Each $p_{i}(k+1 \leq i \leq n)$ receives one share from each of $p_{1}, \ldots, p_{k}$. Thus $c_{i}=i(1 \leq i \leq k)$ and $c_{i}=k(k+1 \leq i \leq n)$. So

$$
s(\mathcal{P})=\frac{1}{2} k(k+1)+k(n-k)=n k-\frac{1}{2} k(k-1) .
$$

We show that the Contraction construction gives an optimal construction for threshold schemes under certain assumptions.

Theorem 5. Let $M$ be an $M T A-f r e e(k, n)$-threshold scheme that is constructed from an ideal unanimous threshold scheme $M_{0}$ on a $k$-subset $A$ of $\mathcal{P}$, and, for cach $p \in A$, a perfect scheme $M_{p}$ for some $\Gamma_{p}$. Then $s(\mathcal{P}) \geq n k-(1 / 2) k(k-1)$.

We now show that if the protocol for establishing an MTA-free scheme is generalised to permit the use of secret sharing schemes that are not perfect then we can always improve on the total storage given by the Contraction construction. Let $0 \leq c \leq k$. A $(c, k, n)$-ramp scheme on an $n$-set $\mathcal{P}$ is a secret sharing scheme such that for $A \subseteq \mathcal{P}$,

1. if $|A| \geq k$ then $H(s \mid A)=0$;

2. if $|A| \leq c$ then $H(s \mid A)=H(s)$.

Ramp schemes such that $H(p)=H(s) /(k-c)(p \in \mathcal{P})$ can be constructed from ideal $(k, n)$-threshold schemes $([7])$.

We now present a construction which works for all $k \leq \frac{1}{2}(n+1)$. This construction relies on implementing the private access structures by using ramp schemes as opposed to perfect threshold schemes. For this reason we call it the Private Ramp construction.

\begin{tabular}{ll} 
& The Private Ramp Construction \\
$\Gamma_{0}$ & $(n, n)$-threshold on $\mathcal{P}$ \\
$\Gamma_{p}$ & $p+((k, n-1)$-threshold on $\mathcal{P} \backslash\{p\})$ \\
$M_{0}$ & ideal $(n, n)$-threshold scheme on $\mathcal{P}$ \\
$M_{p}$ & modified $(0, k, n-1)$-ramp scheme on $\mathcal{P} \backslash\{p\}$ \\
\hline
\end{tabular}

We say that $M_{p}$ is 'modified' because $p \in I_{p}^{\prime}$ and so every distribution rule of $M_{p}$ must also distribute a copy of the secret of $M_{p}$ to participant $p$.

Example 4. Let $\Gamma$ be $(2,3)$-threshold defined on $\mathcal{P}=\{a, b, c\}$. Using the Contraction construction with $\mathcal{P}_{0}=\{a, b\}$ and $\Gamma_{0}=a b$, gives $\Gamma_{a}=a+b c, \Gamma_{b}=b+c$ and a scheme $M_{1}$ for $\Gamma$ with convec $\left(c_{a}, c_{b}, c_{c}\right)=(1,2,2)$. Using the Private Ramp construction gives $\Gamma_{a}=a+b c, \Gamma_{b}=b+a c, \Gamma_{c}=c+a b$. We then use the $(0,2,2)$-ramp schemes $M_{a}, M_{b}, M_{c}$ to construct a scheme $M_{2}$ for $\Gamma$ with convec $(2,2,2)$. Thus $M_{2}$ has a total storage of 6 which is slightly more than the total storage 5 of $M_{1}$. 
Thus for the $(2,3)$ case, the Private Ramp construction did not perform as well as the Contraction construction. In the Private Ramp construction each participant $p \in \mathcal{P}$ generates a share of unit size and then receives $n-1$ other shares, each of size $1 / k$, from the other participants. So for the Private Ramp construction,

$$
s(\mathcal{P})=n\left(1+\frac{n-1}{k}\right)=\frac{n(k+n-1)}{k} .
$$

Thus we can see from (4) and (5) that, generally, the Contraction construction has a lower total storage than the Private Ramp construction when $k$ is small with respect to $n$, but the Private Ramp construction is an improvement on the Contraction construction when $k$ is close to $\frac{1}{2}(n+1)$.

We show now that if a ramp scheme is used to implement the base access structure instead of the private access structures then we can do even better. The Base Ramp construction has the added advantage that it works for all $1 \leq k \leq n-1$.

\section{The Base Ramp Construction}

\begin{tabular}{ll}
$\Gamma_{0}$ & $(n, n)$-threshold on $\mathcal{P}$ \\
$\Gamma_{p}$ & $p+((k, n-1)$-threshold on $\mathcal{P} \backslash\{p\})$ \\
$M_{0}$ & $(k-1, n, n)$-ramp scheme on $\mathcal{P}$ \\
$M_{p}$ & ideal scheme for $\Gamma_{p}(p \in \mathcal{P})$ \\
\hline
\end{tabular}

Example 5. As in Example 4, let $\Gamma$ be $(2,3)$-threshold defined on $\mathcal{P}=\{a, b, c\}$. Using the Base Ramp construction gives $\Gamma_{a}=a+b c, \Gamma_{b}=b+a c, \Gamma_{c}=c+$ $a b$ and a scheme $M_{3}$ for $\Gamma$ with convec $(3 / 2,3 / 2,3 / 2)$. Thus $M_{3}$ has a total storage of $9 / 2$ which is an improvement on the total storage 5 of $M_{1}$ using the Contraction construction, and on the total storage 6 of $M_{2}$ using the Private Ramp construction.

Note that the Base Ramp construction is essentially the same as the Basic construction for a $(k, n)$-threshold scheme, except with a different $M_{0}$. Each participant $p \in \mathcal{P}$ generates a share of size $1 /(n-k+1)$ and then receives $n-1$ other shares, each of size $1 /(n-k+1)$, from the other participants. So for the Base Ramp construction,

$$
s(\mathcal{P})=n\left(\frac{n}{n-k+1}\right)=\frac{n^{2}}{n-k+1} .
$$

Thus from (4) and (6), and from (5) and (6), we see that the Base Ramp scheme is an improvement on both the Contraction construction and the Private Ramp construction (when applicable) for $(k, n)$-threshold schemes. We note that the Base Ramp Construction for threshold schemes can be generalised to other monotone access structures.

We conclude by presenting a table containing the values for the total storage of various MTA-free $(k, n)$-threshold schemes under the constructions discussed 
in this paper ( $*$ denotes that the construction is not possible for these parameters).

\begin{tabular}{|c|c|c|c|c|}
\hline & \multicolumn{4}{|c|}{ Total Storage } \\
\cline { 2 - 5 }$(k, n)$ & Basic & Contraction & Private Ramp & Base Ramp \\
\hline$(2,3)$ & 9 & 5 & 6 & $9 / 2$ \\
$(2,4)$ & 16 & 7 & 10 & $16 / 3$ \\
$(2,5)$ & 25 & 9 & 15 & $25 / 4$ \\
$(3,4)$ & 16 & 9 & $*$ & 8 \\
$(3,5)$ & 25 & 12 & $35 / 3$ & $25 / 3$ \\
$(4,5)$ & 25 & 14 & $*$ & $25 / 2$ \\
$(5,10)$ & 100 & 40 & 28 & $100 / 6$ \\
$(10,20)$ & 400 & 155 & 58 & $400 / 11$ \\
\hline
\end{tabular}

Table 1. Various total storage values for MTA-free $(k, n)$-threshold schemes.

\section{References}

1. J. Benaloh and J. Leichter. Generalized secret sharing and monotone functions. Adv. in Cryptology - CRYPTO'88, Lecture Notes in Comput. Sci, 403:27-35, 1990.

2. G. R. Blakley. Safeguarding cryptographic keys. Proceedings of AFIPS $1979 \mathrm{Na}$ tional Computer Conference, 48:313-317, 1979.

3. E. F. Brickell and D. R. Stinson. Some improved bounds on the information rate of perfect secret sharing schemes. J. Cryptology, 5:153-166, 1992.

4. R. M. Capocelli, A. De Santis, L. Gargano, and U. Vaccaro. On the size of shares for secret sharing schemes. J. Cryptology, 6:157-167, 1993.

5. R. G. Gallager. Information theory and reliable communication. John Wiley and Sons, New York, 1968.

6. I. Ingemarsson and G. J. Simmons. A protocol to set up shared secret schemes without the assistance of a mutually trusted party. Adv. in Cryptology - EURO. CRYPT'90, Lecture Notes in Comput. Sci., 473:266-282, 1991.

7. W.-A. Jackson and K. M. Martin. A combinatorial interpretation of ramp schemes. Submitted, 1994.

8. K. M. Martin. New secret sharing schemes from old. J. Combin. Math Combin. Comput., 14:65-77, 1993.

9. C. Meadows. Some threshold schemes without central key distributors. Congressus Numerantium, 46:187-199, 1985.

10. A. Shamir. How to share a secret. Comm. ACM, 22(11):612-613, 1979.

11. G. J. Simmons. The consequences of trust in shared secret schemes. Adv. in Cryptology - EUROCRYPT'93, Lecture Notes in Compul. Sci., 765:448-452, 1994.

12. G. J. Simmons, W.-A. Jackson, and K. Martin. The geometry of shared secret schemes. Bull. Inst. Combin. Appl., 1:71-88, 1991.

13. D. R. Stinson. An explication of secret sharing schemes. Des. Codes Cryptogr., 2:357-390, 1992.

14. D. R. Stinson. Decomposition constructions for secret sharing schemes. IEEE Trans. Inform. Theory, 40:118-125, 1994. 